\title{
Smoothing Spline Semi-parametric Nonlinear Regression Models
}

\author{
Yuedong Wang and Chunlei Ke*
}

June 23, 2008

\begin{abstract}
We consider the problem of modeling the mean function in regression. Often there is enough knowledge to model some components of the mean function parametrically. But for other vague and/or nuisance components, it is often desirable to leave them unspecified and to be modeled nonparametrically. In this article, we propose a general class of smoothing spline semi-parametric nonlinear regression models (SNRM) which assumes that the mean function depends on parameters and nonparametric functions through a known nonlinear functional. SNRMs are natural extensions of both parametric and nonparametric regression models. They include many popular nonparametric and semi-parametric models such as the partial spline, varying coefficients, projection pursuit, single index, multiple index and shape invariant models as special cases. Building on reproducing kernel Hilbert spaces (RKHS), the SNRMs allow us to deal with many different situations in a unified fashion. We develop a unified estimation procedure based on minimizing penalized likelihood using Gauss-Newton and backfitting algorithms. Smoothing parameters are estimated using the generalized cross-validation (GCV) and generalized maximum likelihood (GML) methods. We derive Bayesian confidence intervals for the unknown functions. A generic and user-friendly R function is developed to implement our estimation and inference procedures. We illustrate our methods
\end{abstract}

\footnotetext{
*Yuedong Wang (email: yuedong@pstat.ucsb.edu) is Professor, Department of Statistics and Applied Probability, University of California, Santa Barbara, California 93106. Chunlei Ke (email: cke@amgen.com) is Statistician, Amgen Inc, One Amgen Center Drive, Thousand Oaks, CA 91320. Yuedong Wang's research was supported by a grant from the National Science Foundation (DMS-0706886). Address for correspondence: Yuedong Wang, Department of Statistics and Applied Probability, University of California, Santa Barbara, California 93106. We thank Dr. Genton for sending us the R Hydrae data. We thank the editor, the associate editor and a referee for constructive comments that substantially improved an earlier draft.
} 
with analyses of three real data sets and evaluate finite-sample performance by simulations.

KEY WORDS: backfitting, Gauss-Newton algorithm, nonlinear functional, nonparametric regression, penalized likelihood, semi-parametric models, smoothing parameter.

\section{Introduction}

Linear and nonlinear parametric regression models are widely used in practice. They use a known function to relate the expected responses to some covariates and a parsimonious set of parameters. Specifically, a nonlinear regression model assumes that (Bates and Watts 1988)

$$
y_{i}=\eta\left(\phi ; \boldsymbol{t}_{i}\right)+\epsilon_{i}, \quad i=1, \cdots, n,
$$

where $\eta$ is a known function of covariates $\boldsymbol{t}_{i}$ and $\phi$ are unknown parameters which provide efficient and interpretable data summary. The form of $\eta$ often comes from scientific theories and/or approximations to mechanics under some simplified assumptions. The specific parametric form of the mean function

may be too restrictive for some applications which could lead to systematic bias and misleading conclusions. In practice, one should always check the form of this function.

It is sometimes difficult, if not impossible, to obtain a specific functional form for $\eta$. Nonparametric regression methods such as smoothing splines (Wahba 1990) provide flexible alternatives in these situations. They do not assume any particular parametric form. Instead, nonparametric regression methods allow data to decide the function. However, nonparametric models lose the advantage of having interpretable parameters.

In this article, we introduce a general class of SNRMs which extends both nonlinear regression and nonparametric regression models. The advantages of combining parametric and nonparametric models have long been recognized. Often in practice there is enough knowledge to model some components in the mean function parametrically. For other vague and/or nuisance components, one may want to leave them unspecified. Many specific semi-parametric models have been proposed in the literature. The simplest semi-parametric model is perhaps the partial linear model which has been studied extensively (Wahba 1990, Härdle, Liang and Gao 2000, Ruppert, Wand and Carroll 2003). Wahba (1990, Ch. 9.1) further proposed the nonlinear partial spline models. Other popular semi-parametric 
models include shape invariant models (Lawton, Sylvestre and Maggio 1972, Wang and Brown 1996), projection pursuit regression (Friedman and Stuetzle 1981), partial linear single and multiple index models (Carroll, Fan, Gijbels and Wand 1997, Yu and Ruppert 2002) and varying-coefficient models (Hastie and Tibshirani 1993). All above models depend on unknown nonparametric functions linearly. Some specific nonlinear models were studied for specific purposes, see for example Ramsay (1998) and Genton and Hall (2007). Mammen and Nielsen (2003) introduced general structure models which are similar to the models proposed in this article. We focus on the development and implementation of methods for estimation and inference. We model nonparametric functions using general smoothing spline models based on RKHS which allows us to deal with functions on different domains in a unified fashion. The SNRMs are extensions of the nonlinear nonparametric regression models in Ke and Wang (2004) by introducing additional parametric components and allowing random errors to be correlated.

In Section 2 we introduce the model and discuss some special cases. We propose estimation and inference methods in Sections 3 and 4 respectively. We present applications of our methods in Section 5 and simulation results in Section 6. This article concludes with summary and discussion of further work in Section 7.

\section{The Model}

We consider the following SNRM

$$
y_{i}=\eta\left(\boldsymbol{\beta}, \boldsymbol{f} ; \boldsymbol{t}_{i}\right)+\epsilon_{i}, \quad i=1, \cdots, n,
$$

where $y_{i}$ 's are observations, $\boldsymbol{t}_{i}=\left(t_{1 i}, \cdots, t_{d i}\right)^{T}$ is a vector of independent variables, $\boldsymbol{\beta}$ is a vector of unknown parameters, $\boldsymbol{f}=\left(f_{1}, \cdots, f_{r}\right)^{T}$ is a vector of unknown functions, and $\boldsymbol{\epsilon}=\left(\epsilon_{1}, \cdots, \epsilon_{n}\right)^{T} \sim$ $N\left(0, \sigma^{2} \boldsymbol{W}^{-1}\right)$. See Pinheiro and Bates (2000) for discussions on possible covariance structures. We do not assume any specific covariance structure. Instead, we assume that $\boldsymbol{W}$ depends on an unknown vector of parameters $\tau$.

We model unknown functions $\boldsymbol{f}$ using general spline models (Wahba 1990). For generality, let the domain of each function $f_{k}, \mathcal{T}_{k}$, be an arbitrary set. We assume that $f_{k} \in \mathcal{H}_{k}$, where $\mathcal{H}_{k}$ is a RKHS on $\mathcal{T}_{k}$. Furthermore, let $\mathcal{H}_{k}=\mathcal{H}_{k}^{0} \oplus \mathcal{H}_{k}^{1}$, where $\mathcal{H}_{k}^{0}=\operatorname{span}\left\{\phi_{k j}, j=1, \cdots, m_{k}\right\}$ is a finite dimensional 
space containing functions which are not penalized, and $\mathcal{H}_{k}^{1}$ is the orthogonal complement of $\mathcal{H}_{k}^{0}$ in $\mathcal{H}_{k} \cdot \mathcal{H}_{k}^{1}$ is a RKHS with reproducing kernel (RK) $R_{k}^{1}$. See Aronszajn (1950), Wahba (1990) and Gu (2002) for details on RKHS. Special spline models include polynomial spline on a continuous interval, periodic spline on the unit circle and thin-plate spline on the Euclidean space. In fact, each domain $\mathcal{T}_{k}$ may be a tensor product of several arbitrary sets and a smoothing spline ANOVA decomposition may be used to model $f_{k}(\mathrm{Gu} 2002)$. For a fixed $\boldsymbol{\beta}, \eta$ acts as a linear or nonlinear functional with respect to $f$.

Our model spaces for $\boldsymbol{f}$ are more general than those in Mammen and Nielsen (2003) which allows us to deal with different situations in a unified fashion (see examples in Section 5). The model space used in Mammen and Nielsen (2003) for the smoothing spline model corresponds to the cubic spline with $\mathcal{T}=[a, b]$ and

$$
\begin{aligned}
\mathcal{H} & =W_{2}([a, b])=\left\{f: f \text { and } f^{\prime} \text { absolutely continuous, } \int_{a}^{b}\left(f^{\prime \prime}\right)^{2} d t<\infty\right\}, \\
\mathcal{H}^{0} & =\operatorname{span}\{1, t\}, \\
R^{1}(s, t) & =\int_{a}^{b}(s-u)_{+}(t-u)_{+} d u,
\end{aligned}
$$

where $W_{2}([a, b])$ is the Sobolev space and $(x)_{+}=\max \{x, 0\}$.

Let $\boldsymbol{y}=\left(y_{1}, \cdots, y_{n}\right)^{T}$ and $\boldsymbol{\eta}(\boldsymbol{\beta}, \boldsymbol{f})=\left(\eta\left(\boldsymbol{\beta}, \boldsymbol{f} ; \boldsymbol{t}_{1}\right), \cdots, \eta\left(\boldsymbol{\beta}, \boldsymbol{f} ; \boldsymbol{t}_{n}\right)\right)^{T}$. Then model $(2)$ can be written in a vector form

$$
\boldsymbol{y}=\boldsymbol{\eta}(\boldsymbol{\beta}, \boldsymbol{f})+\boldsymbol{\epsilon}
$$

When model (2) is linear in $\boldsymbol{f}$ conditional on $\boldsymbol{\beta}, \eta$ can be represented as

$$
\eta\left(\boldsymbol{\beta}, \boldsymbol{f} ; \boldsymbol{t}_{i}\right)=\alpha\left(\boldsymbol{\beta} ; \boldsymbol{t}_{i}\right)+\sum_{k=1}^{r} \mathcal{L}_{k i}(\boldsymbol{\beta}) f_{k},
$$

where $\alpha$ is a known linear or nonlinear function and $\mathcal{L}_{k i}(\boldsymbol{\beta})$ 's are linear functionals depending on $\boldsymbol{\beta}$. Furthermore, when $\mathcal{L}_{k i}$ 's are evaluational functionals, we have

$$
\eta\left(\boldsymbol{\beta}, \boldsymbol{f} ; \boldsymbol{t}_{i}\right)=\alpha\left(\boldsymbol{\beta} ; \boldsymbol{t}_{i}\right)+\sum_{k=1}^{r} \delta_{k}\left(\boldsymbol{\beta} ; \boldsymbol{t}_{i}\right) f_{k}\left(\gamma_{k}\left(\boldsymbol{\beta} ; \boldsymbol{t}_{i}\right)\right),
$$

where $\delta_{k}$ and $\gamma_{k}$ are known functions. Model (6) is interesting in its own right. It covers the most common situations encountered in practice. Nonlinear regression and nonparametric regression models 
are special cases of model (6). Other special cases include partial linear models (Wahba 1990) with $r=$ $1, \boldsymbol{t}=\left(\boldsymbol{t}_{1}^{T}, t_{2}\right)^{T}, \alpha(\boldsymbol{\beta} ; \boldsymbol{t})=\boldsymbol{\beta}^{T} \boldsymbol{t}_{1}, \delta_{1}(\boldsymbol{\beta} ; \boldsymbol{t}) \equiv 1$ and $\gamma_{1}(\boldsymbol{\beta} ; \boldsymbol{t})=t_{2}$; nonlinear partial spline models (Wahba, 1990, Ch. 9.1) with $r=1, \boldsymbol{t}=\left(\boldsymbol{t}_{1}^{T}, t_{2}\right)^{T}, \alpha(\boldsymbol{\beta} ; \boldsymbol{t})=\alpha\left(\boldsymbol{\beta} ; \boldsymbol{t}_{1}\right), \delta_{1}(\boldsymbol{\beta} ; \boldsymbol{t}) \equiv 1$ and $\gamma_{1}(\boldsymbol{\beta} ; \boldsymbol{t})=t_{2}$; partially linear single index model (Carroll et al. 1997, Yu and Ruppert 2002) with $r=1, \alpha(\boldsymbol{\beta} ; \boldsymbol{t})=\boldsymbol{\beta}_{1}^{T} \boldsymbol{t}_{1}$, $\delta_{1}(\boldsymbol{\beta} ; \boldsymbol{t}) \equiv 1$, and $\gamma_{1}(\boldsymbol{\beta} ; \boldsymbol{t})=\boldsymbol{\beta}_{2}^{T} \boldsymbol{t}_{2}$ where $\boldsymbol{\beta}=\left(\boldsymbol{\beta}_{1}^{T}, \boldsymbol{\beta}_{2}^{T}\right)^{T}$ and $\boldsymbol{t}=\left(\boldsymbol{t}_{1}^{T}, \boldsymbol{t}_{2}^{T}\right)^{T}$; varying coefficient models (Hastie and Tibshirani 1993) with $\alpha(\boldsymbol{\beta} ; \boldsymbol{t})=\beta_{1}, \delta_{k}(\boldsymbol{\beta} ; \boldsymbol{t})=x_{k}$ for a known predictor $x_{k}$ which could depend on $\boldsymbol{t}$ and $\gamma_{k}(\boldsymbol{\beta} ; \boldsymbol{t})=t_{k}$; and project pursuit regression model (Friedman and Stuetzle 1981) with $\alpha(\boldsymbol{\beta} ; \boldsymbol{t})=\beta_{0}, \delta_{k}(\boldsymbol{\beta} ; \boldsymbol{t}) \equiv 1$ and $\gamma_{k}(\boldsymbol{\beta} ; \boldsymbol{t})=\boldsymbol{\beta}_{k}^{T} \boldsymbol{t}$ where $\boldsymbol{\beta}=\left(\beta_{0}, \boldsymbol{\beta}_{1}^{T}, \cdots, \boldsymbol{\beta}_{r}^{T}\right)^{T}$.

Grouped data are common in practice. They include repeated measures, longitudinal, functional and multilevel data as special cases. We define SNRM for grouped data as

$$
y_{i j}=\eta\left(\boldsymbol{\beta}_{i}, \boldsymbol{f} ; \boldsymbol{t}_{i j}\right)+\epsilon_{i j}, \quad i=1, \cdots, m, \quad j=1, \cdots, n_{i}
$$

where $y_{i j}$ is the response of experimental unit $i$ at design point $\boldsymbol{t}_{i j}, \boldsymbol{t}_{i j}=\left(t_{1 i j}, \cdots, t_{d i j}\right)$ is a vector of independent variables, $\eta$ is a known function of $\boldsymbol{t}_{i j}$ which depends on a vector of parameter $\boldsymbol{\beta}_{i}$ and a vector of unknown nonparametric functions $\boldsymbol{f}$, and $\boldsymbol{\epsilon}=\left(\epsilon_{11}, \cdots, \epsilon_{1 n_{1}}, \cdots, \epsilon_{m 1}, \cdots \epsilon_{m n_{m}}\right)^{T} \sim$ $\mathrm{N}\left(0, \sigma^{2} \boldsymbol{W}^{-1}\right)$. The shape invariant model in Lawton et al. (1972) is a special case of model (7) with $d=1, r=1, \eta\left(\boldsymbol{\beta}_{i}, f ; t_{i j}\right)=\beta_{i 1}+\beta_{i 2} f\left(\left(t_{i j}-\beta_{i 3}\right) / \beta_{i 4}\right)$. Let $n=\sum_{i=1}^{m} n_{i}, \boldsymbol{y}_{i}=\left(y_{i 1}, \cdots, y_{i n_{i}}\right)^{T}$, $\boldsymbol{y}=\left(\boldsymbol{y}_{1}^{T}, \cdots, \boldsymbol{y}_{m}^{T}\right)^{T}, \boldsymbol{\eta}_{i}\left(\boldsymbol{\beta}_{i}, \boldsymbol{f}\right)=\left(\eta\left(\boldsymbol{\beta}_{i}, \boldsymbol{f} ; \boldsymbol{t}_{i 1}\right), \cdots, \eta\left(\boldsymbol{\beta}_{i}, \boldsymbol{f} ; \boldsymbol{t}_{i n_{i}}\right)\right)^{T}, \boldsymbol{\beta}=\left(\boldsymbol{\beta}_{1}^{T}, \cdots, \boldsymbol{\beta}_{m}^{T}\right)^{T}$, and $\boldsymbol{\eta}(\boldsymbol{\beta}, \boldsymbol{f})=$ $\left(\boldsymbol{\eta}_{1}^{T}\left(\boldsymbol{\beta}_{1}, \boldsymbol{f}\right), \cdots, \boldsymbol{\eta}_{m}^{T}\left(\boldsymbol{\beta}_{m}, \boldsymbol{f}\right)\right)^{T}$. Then model (7) can be written in the same vector form as (4).

Remark 1. Parameters $\boldsymbol{\beta}$ may depend on other covariates which can be built into a second stage model as in Pinheiro and Bates (2000). For simplicity, we do not assume a second stage model in this article even though our proposed estimation methods and software also apply to these models. Also for simplicity, we will present estimation and inference methods for model (2) only. Methods for model (7) for grouped data are similar.

The mean function $\eta$ may depend on both parameters $\boldsymbol{\beta}$ and nonparametric functions $\boldsymbol{f}$ nonlinearly. The nonparametric functions $\boldsymbol{f}$ are regarded as parameters, just like $\boldsymbol{\beta}$. Certain constraints may be required to make a SNRM identifiable. Specific conditions depend on the form of a model and the purpose of an analysis. Often identifiability can be achieved by absorbing some parameters into $f$ 
and/or adding constraints on $\boldsymbol{f}$ by removing certain components from the model spaces.

\section{Estimation}

\subsection{Penalized Likelihood}

We shall estimate the unknown functions $\boldsymbol{f}$ as well as parameters $\boldsymbol{\beta}, \boldsymbol{\tau}$ and $\sigma^{2}$. We estimate $\boldsymbol{\beta}, \boldsymbol{\tau}$ and $f$ as minimizers of the following penalized likelihood

$$
-\log L\left(\boldsymbol{y} ; \boldsymbol{\beta}, \boldsymbol{f}, \boldsymbol{\tau}, \sigma^{2}\right)+\frac{n \lambda}{2 \sigma^{2}} \sum_{k=1}^{r} \theta_{k}^{-1}\left\|P_{k 1} f_{k}\right\|^{2},
$$

where $L\left(\boldsymbol{y} ; \boldsymbol{\beta}, \boldsymbol{f}, \boldsymbol{\tau}, \sigma^{2}\right)$ is the likelihood function of $\boldsymbol{y}, P_{k 1}$ is the projection operator onto the subspace $\mathcal{H}_{k}^{1}$ in $\mathcal{H}_{k},\left\|P_{k 1} f_{k}\right\|^{2}$ is a penalty to the departure from the null space $\mathcal{H}_{k}^{0}$, and $\lambda$ and $\boldsymbol{\theta}=\left(\theta_{1}, \cdots, \theta_{r}\right)$ are smoothing parameters balancing the trade-off between goodness-of-fit and penalties. Selection options for the model space $\mathcal{H}_{k}$, its decomposition $\mathcal{H}_{k}=\mathcal{H}_{k}^{0} \oplus \mathcal{H}_{k}^{1}$ and the penalty $\left\|P_{k 1} f_{k}\right\|^{2}$ provide flexibility to construct different models based on prior knowledge, identifiability constraints and purpose of the study. See Section 5 for examples. If $f_{k}$ is multivariate, then $\left\|P_{k 1} f_{k}\right\|^{2}$ may include more than one penalty terms such as those used in smoothing spline ANOVA models (Wahba 1990, Gu 2002).

Given $\boldsymbol{\beta}$ and $\boldsymbol{\tau}$, the solutions of $\boldsymbol{f}$ to (8) exist and are unique under certain regularity conditions (Ke and Wang 2004). In general, the solutions of $\boldsymbol{f}$ may not lie in finite dimensional spaces. Therefore certain approximations are necessary. Throughout this article we assume that the solutions of $\boldsymbol{f}$ exist.

Different methods have been proposed to minimize (8) for some special cases of the SNRM. Estimation for the general SNRM is challenging since functions $\boldsymbol{f}$ may interact with $\boldsymbol{\beta}$ and $\boldsymbol{\tau}$ in a complicated manner. We will develop an estimation procedure for model (5) first and then extend the procedure to the nonlinear case.

\subsection{Estimation for Model (5)}

Under model (5) where $\eta$ depends on $\boldsymbol{f}$ linearly, for fixed $\boldsymbol{\beta}$ and $\boldsymbol{\tau}$, the solutions of $\boldsymbol{f}$ to (8) are in finite dimensional spaces and can be represented by (Wahba 1990)

$$
\hat{f}_{k}\left(\boldsymbol{\beta}, \boldsymbol{\tau} ; t_{k}\right)=\sum_{v=1}^{m_{k}} d_{k v} \phi_{k v}\left(t_{k}\right)+\theta_{k} \sum_{i=1}^{n} c_{i} \xi_{k i}\left(t_{k}\right),
$$


where $\xi_{k i}\left(t_{k}\right)=\mathcal{L}_{k i(\cdot)} R_{k}^{1}\left(t_{k}, \cdot\right)$. We need to solve coefficients $\boldsymbol{d}=\left(d_{11}, \cdots, d_{1 m_{1}}, \cdots, d_{r 1}, \cdots, d_{r m_{r}}\right)^{T}$ and $\boldsymbol{c}=\left(c_{1}, \cdots, c_{n}\right)^{T}$ as minimizers of the penalized likelihood (8).

Let $\boldsymbol{T}_{k}=\left\{\mathcal{L}_{k i} \phi_{k v}\right\}_{i=1 v=1}^{n} m_{k}, k=1, \cdots, r$, and $\boldsymbol{T}=\left(\boldsymbol{T}_{1}, \cdots, \boldsymbol{T}_{r}\right)$. $\boldsymbol{T}$ is a $n \times m$ matrix with $m=$ $\sum_{k=1}^{r} m_{k}$. We assume that $\boldsymbol{T}$ is of full column rank. Let $\boldsymbol{\Sigma}_{k}=\left\{\mathcal{L}_{k i} \mathcal{L}_{k j} R_{k}^{1}\right\}_{i, j=1}^{n}$ and $\boldsymbol{\Sigma}=\sum_{k=1}^{r} \theta_{k} \boldsymbol{\Sigma}_{k}$. Note that both $\boldsymbol{T}$ and $\boldsymbol{\Sigma}$ may depend on $\boldsymbol{\beta}$ even though the dependence is not explicitly expressed for the simplicity of notations. Plugging (9) back in (8), we have

$$
\log \left|\sigma^{2} \boldsymbol{W}^{-1}\right|+\frac{1}{\sigma^{2}}(\boldsymbol{y}-\boldsymbol{\alpha}-\boldsymbol{T} \boldsymbol{d}-\boldsymbol{\Sigma} \boldsymbol{c})^{T} \boldsymbol{W}(\boldsymbol{y}-\boldsymbol{\alpha}-\boldsymbol{T} \boldsymbol{d}-\boldsymbol{\Sigma} \boldsymbol{c})+\frac{n \lambda}{\sigma^{2}} \boldsymbol{c}^{T} \boldsymbol{\Sigma} \boldsymbol{c}
$$

where $\boldsymbol{\alpha}=\left(\alpha\left(\boldsymbol{\beta} ; \boldsymbol{t}_{1}\right), \cdots, \alpha\left(\boldsymbol{\beta} ; \boldsymbol{t}_{n}\right)\right)^{T}$. Let $\tilde{\boldsymbol{y}}=W^{1 / 2}(\boldsymbol{y}-\boldsymbol{\alpha}), \tilde{\boldsymbol{T}}=W^{1 / 2} \boldsymbol{T}, \tilde{\boldsymbol{\Sigma}}=W^{1 / 2} \boldsymbol{\Sigma} W^{1 / 2}, \tilde{\boldsymbol{c}}=$ $W^{-1 / 2} \boldsymbol{c}$, and $\tilde{\boldsymbol{d}}=\boldsymbol{d}$. Ignoring constants independent of $\boldsymbol{c}$ and $\boldsymbol{d},(10)$ is equivalent to

$$
\|\tilde{\boldsymbol{y}}-\tilde{\boldsymbol{T}} \tilde{\boldsymbol{d}}-\tilde{\boldsymbol{\Sigma}} \tilde{\boldsymbol{c}}\|^{2}+n \lambda \tilde{\boldsymbol{c}}^{T} \tilde{\boldsymbol{\Sigma}} \tilde{\boldsymbol{c}} .
$$

Solutions to (11) are given in Wahba (1990). Coefficients $\tilde{\boldsymbol{c}}$ and $\tilde{\boldsymbol{d}}$ can be solved by software packages such as RKPACK (Gu 1989). Then solutions to (10) are $\boldsymbol{d}=\tilde{\boldsymbol{d}}$ and $\boldsymbol{c}=W^{1 / 2} \tilde{\boldsymbol{c}}$. Let $\tilde{\boldsymbol{M}}=\tilde{\boldsymbol{\Sigma}}+n \lambda \boldsymbol{I}$ where $\boldsymbol{I}$ is the identity matrix. It is known that $\tilde{\boldsymbol{T}} \tilde{\boldsymbol{d}}+\tilde{\boldsymbol{\Sigma}} \tilde{\boldsymbol{c}}=\tilde{\boldsymbol{A}} \tilde{\boldsymbol{y}}$, where

$$
\tilde{\boldsymbol{A}}=n \lambda \tilde{\boldsymbol{M}}^{-1}\left\{\boldsymbol{I}-\tilde{\boldsymbol{T}}\left(\tilde{\boldsymbol{T}}^{T} \tilde{\boldsymbol{M}}^{-1} \tilde{\boldsymbol{T}}\right)^{-1} \tilde{\boldsymbol{T}}^{T} \tilde{\boldsymbol{M}}^{-1}\right\}
$$

is the hat matrix (Wahba 1990).

Smoothing parameters are crucial for the performance of spline estimates. Many data-driven procedures have been developed for estimating smoothing parameters. The GCV and GML methods are widely used for estimating smoothing parameters due to their good performances. The GCV and GML estimates of smoothing parameters are minimizers of (Wahba 1990, Gu 2002)

$$
\begin{aligned}
V(\lambda, \boldsymbol{\theta}) & =\frac{\frac{1}{n}\|(\boldsymbol{I}-\tilde{\boldsymbol{A}}) \tilde{\boldsymbol{y}}\|^{2}}{\left\{\frac{1}{n} \operatorname{tr}(\boldsymbol{I}-\tilde{\boldsymbol{A}})\right\}^{2}}, \\
M(\lambda, \boldsymbol{\theta}) & =\frac{\tilde{\boldsymbol{y}}^{T}(\boldsymbol{I}-\tilde{\boldsymbol{A}}) \tilde{\boldsymbol{y}}}{\left\{\operatorname{det}^{+}(\boldsymbol{I}-\tilde{\boldsymbol{A}})\right\}^{1 /(n-m)}},
\end{aligned}
$$

where $\operatorname{det}^{+}$is the product of nonzero eigenvalues.

We use the backfitting procedure to estimate $\boldsymbol{f}$ and parameters $\boldsymbol{\beta}$ and $\boldsymbol{\tau}$ alternatively. Given the current estimates of $\boldsymbol{\beta}$ and $\boldsymbol{\tau}$, say $\boldsymbol{\beta}_{-}$and $\boldsymbol{\tau}_{-}$, we use the above procedure to obtain new estimates of 
$\boldsymbol{f}, \hat{\boldsymbol{f}}_{-}\left(\boldsymbol{\beta}_{-}, \boldsymbol{\tau}_{-}\right)$. Given the current estimate of $\boldsymbol{f}$, we obtain new estimates of $\boldsymbol{\beta}$ and $\boldsymbol{\tau}$ by minimizing negative log-likelihood

$$
\log \left|\sigma^{2} \boldsymbol{W}^{-1}\right|+\frac{1}{\sigma^{2}}\left\{\boldsymbol{y}-\boldsymbol{\eta}\left(\boldsymbol{\beta}, \hat{\boldsymbol{f}}_{-}\left(\boldsymbol{\beta}_{-}, \boldsymbol{\tau}_{-}\right)\right)\right\}^{T} \boldsymbol{W}\left\{\boldsymbol{y}-\boldsymbol{\eta}\left(\boldsymbol{\beta}, \hat{\boldsymbol{f}}_{-}\left(\boldsymbol{\beta}_{-}, \boldsymbol{\tau}_{-}\right)\right)\right\} .
$$

Optimization methods such as the Newton-Raphson procedure can be used to solve (15). We adopt the backfitting and Gauss-Newton algorithms in Pinheiro and Bates (2000) (Section 7.5.2) to solve (15) by updating $\boldsymbol{\beta}$ and $\boldsymbol{\tau}$ iteratively. Putting pieces together, we have the following algorithm.

\section{Algorithm 1}

1. Set initial values for $\boldsymbol{\beta}$ and $\boldsymbol{\tau}$;

2. Cycle between (a) and (b) until convergence:

(a) conditional on current estimates of $\boldsymbol{\beta}$ and $\boldsymbol{\tau}$, update $\boldsymbol{f}$ by solving (11) with smoothing parameters selected by the GCV or GML method;

(b) conditional on current estimates of $\boldsymbol{f}$, update $\boldsymbol{\beta}$ and $\boldsymbol{\tau}$ by solving (15) alternatively using the backfitting and Gauss-Newton algorithms in Pinheiro and Bates (2000).

Remark 2. As in Wahba, Wang, Gu, Klein and Klein (1995), smoothing parameters are estimated iteratively with fixed $\boldsymbol{W}$ at step 2(a). The GCV and GML criteria are different from those in Wang (1998) where the covariance parameters $\boldsymbol{\tau}$ are estimated together with smoothing parameters. We estimate $\boldsymbol{\tau}$ at step 2(b) since it is easier to implement and is computationally less expensive.

Denote the final estimates of $\boldsymbol{\beta}, \boldsymbol{\tau}$ and $\boldsymbol{f}$ as $\hat{\boldsymbol{\beta}}, \hat{\boldsymbol{\tau}}$ and $\hat{\boldsymbol{f}}$. We estimate $\sigma^{2}$ by

$$
\hat{\sigma}^{2}=\frac{(\boldsymbol{y}-\hat{\boldsymbol{\eta}})^{T} \hat{\boldsymbol{W}}(\boldsymbol{y}-\hat{\boldsymbol{\eta}})}{n-p-\operatorname{tr}\left(\tilde{\boldsymbol{A}}^{*}\right)},
$$

where $\hat{\boldsymbol{\eta}}=\boldsymbol{\eta}(\hat{\boldsymbol{\beta}}, \hat{\boldsymbol{f}})$ and $\hat{\boldsymbol{W}}$ is the estimate of $\boldsymbol{W}$ with $\boldsymbol{\tau}$ replaced by $\hat{\boldsymbol{\tau}}, p$ is the degrees of freedom for parameters which is usually taken as the total number of parameters and $\tilde{\boldsymbol{A}}^{*}$ is the hat matrix (12) computed at convergence.

\subsection{Extended Gauss-Newton Procedure}

If $\eta$ is nonlinear in $\boldsymbol{f}$, the solutions of $\boldsymbol{f}$ to (8) are not in finite dimensional spaces and thus certain approximations have to be made. One approach is to approximate each $f_{k}$ using a finite collection of 
basis functions or representers. One then needs to select the basis functions or representers which may become difficult when the domain of the function is multivariate. We adopt an alternative approach which is fully adaptive and data-driven.

Let $\boldsymbol{f}_{-}$be the current estimate of $\boldsymbol{f}$. For any fixed $\boldsymbol{\beta}, \eta$ is a functional from $\mathcal{H}_{1} \times \cdots \times \mathcal{H}_{r}$ to $R$. We assume that the Fréchet differential of $\eta$ with respect to $\boldsymbol{f}$ evaluated at $\boldsymbol{f}_{-}, \mathcal{L}_{i}=\left.D \eta\left(\boldsymbol{\beta}, \boldsymbol{f} ; \boldsymbol{t}_{i}\right)\right|_{\boldsymbol{f}=\boldsymbol{f}^{-}}$, exist and is bounded (Flett 1980). Then $\mathcal{L}_{i} \boldsymbol{h}=\sum_{k=1}^{r} \mathcal{L}_{k i} h_{k}$ where $\mathcal{L}_{k i}$ is the partial Fréchet differential of $\eta$ with respect to $f_{k}$ evaluated at $\boldsymbol{f}_{-}$and $h_{k} \in \mathcal{H}_{k}$ (Flett, 1980, Ch. 3.3). $\mathcal{L}_{k i}$ is a bounded linear functional from $\mathcal{H}_{k}$ to $R$. Approximate $\eta$ in (2) by its first order Taylor expansion at $\boldsymbol{f}^{-}$(Flett 1980)

$$
\eta\left(\boldsymbol{\beta}, \boldsymbol{f} ; \boldsymbol{t}_{i}\right) \approx \eta\left(\boldsymbol{\beta}, \boldsymbol{f}_{-} ; \boldsymbol{t}_{i}\right)+\sum_{k=1}^{r} \mathcal{L}_{k i}\left(f_{k}-f_{k-}\right)=\alpha\left(\boldsymbol{\beta} ; \boldsymbol{t}_{i}\right)+\sum_{k=1}^{r} \mathcal{L}_{k i} f_{k},
$$

where

$$
\alpha\left(\boldsymbol{\beta} ; \boldsymbol{t}_{i}\right)=\eta\left(\boldsymbol{\beta}, \boldsymbol{f}_{-} ; \boldsymbol{t}_{i}\right)-\sum_{k=1}^{r} \mathcal{L}_{k i} f_{k-} .
$$

The approximated mean function (17) is in the form of (5) which leads to the following algorithm.

\section{Algorithm 2}

1. Set initial values for $\boldsymbol{\beta}, \boldsymbol{\tau}$ and $\boldsymbol{f}$;

2. Cycle between (a) and (b) until convergence:

(a) conditional on current estimates of $\boldsymbol{\beta}$ and $\boldsymbol{\tau}$, compute $\mathcal{L}_{k i}$ and $\alpha\left(\boldsymbol{\beta} ; \boldsymbol{t}_{i}\right)$ in (18), and update $\boldsymbol{f}$ using step 2(a) in Algorithm 1 based on the approximate mean function (17). Repeat this step until convergence;

(b) conditional on current estimates of $\boldsymbol{f}$, update $\boldsymbol{\beta}$ and $\boldsymbol{\tau}$ by solving (15) alternatively using the backfitting and Gauss-Newton algorithms in Pinheiro and Bates (2000).

Step 2(a) in the above algorithm is an extension of the Gauss-Newton method to an infinite dimensional space. 


\section{Inference}

Inference for $\boldsymbol{\beta}$ and $\boldsymbol{\tau}$ can be made based on the approximate distributions of the maximum likelihood estimates (Pinheiro and Bates 2000). For smoothing spline models, Bayesian confidence intervals are often used to assess the spline estimates (Wahba 1990, Gu 2002). We now construct Bayesian confidence intervals for $\boldsymbol{f}$ in SNRMs. $\boldsymbol{\beta}$ and $\boldsymbol{\tau}$ are fixed in the following arguments.

For standard smoothing spline models, Wahba (1978) showed that spline smoothing is equivalent to Bayesian estimation. We now extend this connection to SNRMs. Assume priors for $f_{k}$ 's as

$$
F_{k}\left(t_{k}\right)=\sum_{\nu=1}^{m_{k}} \zeta_{k \nu} \phi_{k \nu}\left(t_{k}\right)+\sqrt{b \theta_{k}} X_{k}\left(t_{k}\right), \quad k=1, \cdots, r
$$

where $\boldsymbol{\zeta}=\left(\zeta_{11}, \cdots, \zeta_{1 m_{1}}, \cdots, \zeta_{r 1}, \cdots, \zeta_{r m_{r}}\right) \stackrel{i i d}{\sim} \mathrm{N}(0, a \boldsymbol{I}), X_{k}$ 's are independent zero mean Gaussian stochastic processes with $\mathrm{E}\left\{X_{k}\left(s_{k}\right) X_{k}\left(t_{k}\right)\right\}=R_{k}^{1}\left(s_{k}, t_{k}\right)$, and $\zeta$ and $X_{k}$ 's are independent. We will consider the model (5) first and, without loss of generality, we assume that $\boldsymbol{\alpha}=0$ since otherwise it can be absorbed into $\boldsymbol{y}$. Suppose that observations are generated by

$$
y_{i}=\sum_{k=1}^{r} \mathcal{L}_{k i} F_{k}+\epsilon_{i}, \quad i=1, \cdots, n .
$$

See Wahba (1990, Ch. 1.4) for the definition of $\mathcal{L}_{k i} X_{k}$. Since model (20) involves linear functionals, following similar arguments as in Wahba (1990), it can be shown that with $b=\sigma^{2} / n \lambda$,

$$
\lim _{a \rightarrow \infty} \mathrm{E}\left\{F_{k}\left(t_{k}\right) \mid \boldsymbol{y}\right\}=\hat{f}_{k}\left(t_{k}\right), \quad k=1, \cdots, r .
$$

Therefore, the spline estimates are the same as the posterior means.

To compute Bayesian confidence intervals for components in functions $f_{k}$, let $g_{j \nu}^{0}\left(s_{j}\right)=\zeta_{j \nu} \phi_{j \nu}\left(s_{j}\right)$, $g_{k \mu}^{0}\left(t_{k}\right)=\zeta_{k \mu} \phi_{k \mu}\left(t_{k}\right), g_{j}\left(s_{j}\right)=\sqrt{b \theta_{j}} X_{j}\left(s_{j}\right)$ and $g_{k}\left(t_{k}\right)=\sqrt{b \theta_{k}} X_{k}\left(t_{k}\right), j=1, \cdots, r, k=1, \cdots, r$, $\nu=1, \cdots, m_{j}, \mu=1, \cdots, m_{k}$. It is not difficult to show that results in Gu and Wahba (1993) hold 
for model (5) with general linear operators and correlated errors (Appendix). Specifically, we have

$$
\begin{aligned}
\mathrm{E}\left\{g_{k \mu}^{0}\left(t_{k}\right) \mid \boldsymbol{y}\right\} & =d_{k \mu} \phi_{k \mu}\left(t_{k}\right), \\
\mathrm{E}\left\{g_{k}\left(t_{k}\right) \mid \boldsymbol{y}\right\} & =\theta_{k} \sum_{i=1}^{n} c_{i} \xi_{k i}\left(t_{k}\right), \\
b^{-1} \operatorname{Cov}\left\{g_{j \nu}^{0}\left(s_{j}\right), g_{k \mu}^{0}\left(t_{k}\right) \mid \boldsymbol{y}\right\} & =\phi_{j \nu}\left(s_{j}\right) \phi_{k \mu}\left(t_{k}\right) u_{j \nu k \mu}, \\
b^{-1} \operatorname{Cov}\left\{g_{j}\left(s_{j}\right), g_{k \mu}^{0}\left(t_{k}\right) \mid \boldsymbol{y}\right\} & =-v_{j \mu}\left(s_{j}\right) \phi_{k \mu}\left(t_{k}\right), \\
b^{-1} \operatorname{Cov}\left\{g_{k}\left(s_{k}\right), g_{k}\left(t_{k}\right) \mid \boldsymbol{y}\right\} & =\theta_{k} R_{k}^{1}\left(s_{k}, t_{k}\right)-\theta_{k} \sum_{i=1}^{n} w_{k i} \xi_{k i}\left(t_{k}\right), \\
b^{-1} \operatorname{Cov}\left\{g_{j}\left(s_{j}\right), g_{k}\left(t_{k}\right) \mid \boldsymbol{y}\right\} & =-\theta_{k} \sum_{i=1}^{n} w_{j i} \xi_{k i}\left(t_{k}\right), \quad j \neq k,
\end{aligned}
$$

where $u_{j \nu k \mu}$ is the $\left(\sum_{l=1}^{j-1} m_{l}+\nu, \sum_{l=1}^{k-1} m_{l}+\mu\right)$ th element of the matrix $\left(\boldsymbol{T}^{T} \boldsymbol{M}^{-1} \boldsymbol{T}\right)^{-1}, v_{j \mu}\left(s_{j}\right)$ is the $\left(\sum_{l=1}^{j-1} m_{l}+\mu\right)$ th element of the vector $\theta_{j}\left(\boldsymbol{T}^{T} \boldsymbol{M}^{-1} \boldsymbol{T}\right)^{-1} \boldsymbol{T}^{T} \boldsymbol{M}^{-1} \boldsymbol{\xi}_{j}\left(s_{j}\right), w_{j i}$ is the $i$ th element of $\theta_{j}\left\{\boldsymbol{M}^{-1}-\boldsymbol{M}^{-1} \boldsymbol{T}\left(\boldsymbol{T}^{T} \boldsymbol{M}^{-1} \boldsymbol{T}\right)^{-1} \boldsymbol{T}^{T} \boldsymbol{M}^{-1}\right\} \boldsymbol{\xi}_{j}\left(s_{j}\right), \boldsymbol{M}=\boldsymbol{\Sigma}+n \lambda \boldsymbol{W}^{-1}$ and $\boldsymbol{\xi}_{j}\left(s_{j}\right)=\left(\xi_{j 1}\left(s_{j}\right), \cdots, \xi_{j n}\left(s_{j}\right)\right)^{T}$. For simplicity, $\sum_{l=1}^{0} m_{l}=0$.

The first two equations in (21) state that the projections of $\hat{f}_{k}$ on subspaces are the posterior means of the corresponding components in the Bayesian model (19). The next four equations in (21) can be used to compute posterior covariances of the spline estimates and their projections. Computational methods in Gu and Wahba (1993) are modified to compute posterior covariances. Based on these posterior covariances, we construct Bayesian confidence intervals for the smoothing spline estimates $\hat{f}_{k}$ and their components as Gu and Wahba (1993).

For the general SNRM, when $\eta$ is nonlinear in $\boldsymbol{f}$, we can approximate model (2) based on the first order Taylor expansion of $\eta(\boldsymbol{\beta}, \boldsymbol{f})$ at $\hat{\boldsymbol{f}}$

$$
\tilde{y}_{i}=\sum_{k=1}^{r} \mathcal{L}_{k i} f_{k}+\epsilon_{i}, \quad i=1, \cdots, n,
$$

where $\tilde{y}_{i}=y_{i}-\eta\left(\boldsymbol{\beta}, \hat{\boldsymbol{f}} ; \boldsymbol{t}_{i}\right)+\sum_{k=1}^{r} \mathcal{L}_{k i} \hat{f}_{k}$ and $\mathcal{L}_{k i}$ is the partial Fréchet differential of $\eta$ with respect to $f_{k}$ evaluated at $\hat{\boldsymbol{f}}$. Posterior covariances for model (22) can be calculated using formulae in (21) which provide approximate posterior covariances for the original model. Bayesian confidence intervals are then constructed based on these approximate posterior covariances.

Bootstrap is an alternative approach to construct confidence intervals for $f$. A bootstrap sample 
is generated as

$$
y_{i}^{*}=\eta\left(\hat{\boldsymbol{\beta}}, \hat{\boldsymbol{f}} ; \boldsymbol{t}_{i}\right)+\epsilon_{i}^{*}, \quad i=1, \cdots, n,
$$

where $\epsilon^{*}=\left(\epsilon_{1}^{*}, \cdots, \epsilon_{n}^{*}\right)^{T}$ are random sample from $N\left(0, \hat{\sigma}^{2} \hat{\boldsymbol{W}}^{-1}\right)$ or residuals. The SNMR is fitted to the bootstrap sample. This procedure is repeated for a number of times and bootstrap confidence intervals are then constructed as in Wang and Wahba (1995). For standard smoothing spline models, it is known that the performance of Bayesian and bootstrap confidence intervals are comparable and both have the "across-the-function" coverage property (Wang and Wahba 1995). Bootstrap method is more computationally intensive. We will investigate their performance in Section 6 .

\section{Application}

In this section, to illustrate versatility of the general methodology, we fit several SNRMs to three real datasets. The GCV or GML method are used to estimate smoothing parameters in all examples. They usually lead to similar results.

\subsection{The Evolution of the Mira Variable R Hydrae}

The Mira variable R Hydrae is well known for its declining period and amplitude (Zijlstra, Bedding and Mattei 2002). It is of great interest to investigate the pattern of the decline. Genton and Hall (2007) proposed the following semi-parametric model

$$
y_{i}=a\left(t_{i}\right) f_{1}\left(x\left(t_{i}\right)\right)+\epsilon_{i}, \quad i=1, \cdots, n
$$

where $y_{i}$ is observation of the magnitude (brightness) at time $t_{i}, a(t)$ is the amplitude function, $f_{1}$ is the common periodic shape function with unit period, $x(t)$ is a strictly increasing time transformation function and $\epsilon_{i}$ are iid random errors with mean zero and variance $\sigma^{2} .1 / x^{\prime}(t)$ can be regarded as the periodic. Genton and Hall (2007) modeled $f_{1}$ nonparametrically using kernel method and suggested to model $a(t)$ and $x(t)$ parametrically. In particular, Genton and Hall (2007) fitted observations during 1900-1950 ( $n=2315)$ with linearly evolving amplitude and period

$$
\begin{aligned}
& a(t)=1+\omega_{1} t, \\
& x(t)=\theta_{2}^{-1} \log \left(1+\theta_{1}^{-1} \theta_{2} t\right) .
\end{aligned}
$$


They obtained estimates $\hat{\omega}_{1}=3.964 \times 10^{-7}, \hat{\theta}_{1}=419.3$ and $\hat{\theta}_{2}=-1.441 \times 10^{-3}$. The kernel estimate of $f_{1}$ is shown in Figure 1. The estimated amplitude function is flat.
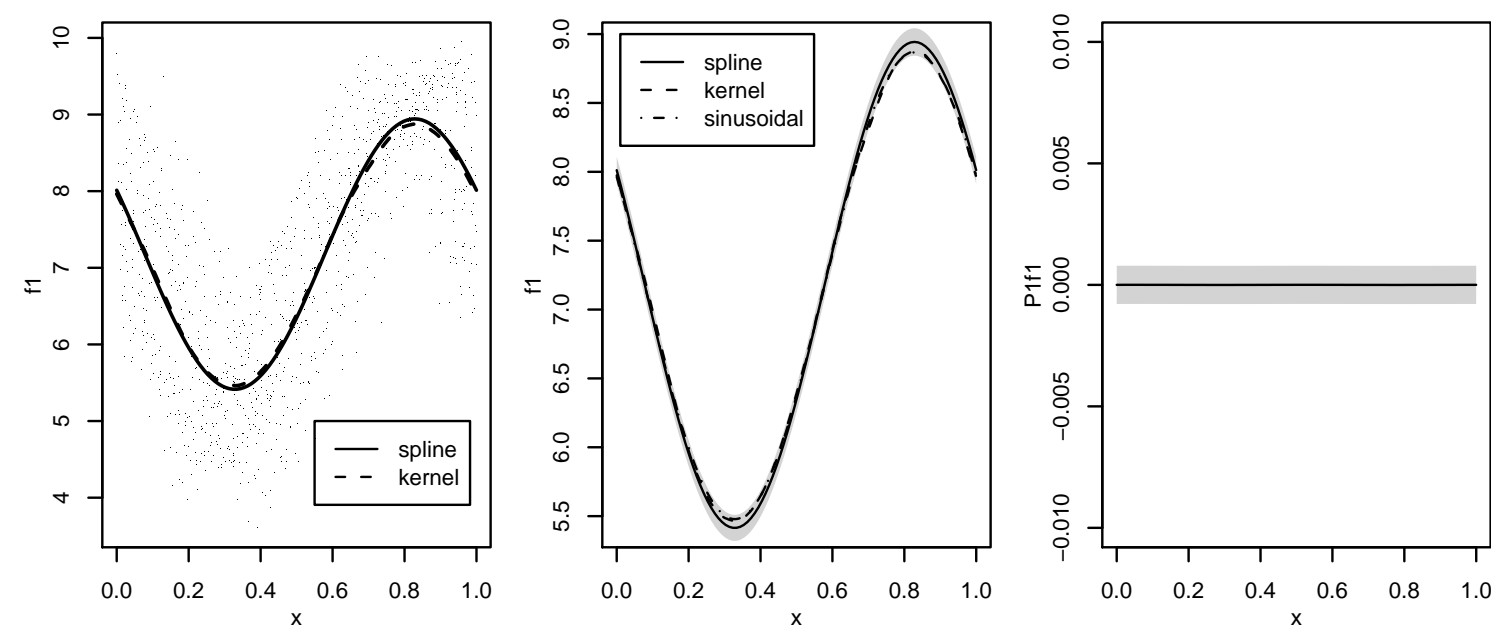

Figure 1: Left: folded plot with nonparametric estimates of $f_{1}$. Middle: estimates of $f_{1}$ and $95 \%$ Bayesian confidence intervals for the spline estimate. Right: projection of the spline estimate onto space $\mathcal{H}^{1}$ and its $95 \%$ Bayesian confidence intervals.

Model (23) is a special case of the SNRM. We fit model (23) with the same parametric models (24) for amplitude and (25) for period. We model $f_{1}$ using periodic spline model space (Gu 2002)

$$
W_{2}(\text { per })=\left\{f: f \text { and } f^{\prime} \text { are abs. cont., } f(0)=f(1), f^{\prime}(0)=f^{\prime}(1), \int_{0}^{1}\left(f^{\prime \prime}(t)\right)^{2} d t<\infty\right\} .
$$

Since $f_{1}$ is close to a sinusoidal function, instead of the usual roughness penalty, we use the L-spline with the penalty $\left\|P_{1} f_{1}\right\|^{2}=\int_{0}^{1}\left(L f_{1}\right)^{2} d t$ where $L=D\left\{D^{2}+(2 \pi)^{2}\right\}$, and $D^{j}$ is the $j$ th derivative operator. $W_{2}($ per $)=\mathcal{H}^{0} \oplus \mathcal{H}^{1}$ where $\mathcal{H}^{0}=\operatorname{span}\{1, \sin 2 \pi t, \cos 2 \pi t\}, \mathcal{H}^{1}$ is a RKHS with RK $R^{1}(s, t)=$ $\sum_{\nu=2}^{\infty} 2 \cos 2 \pi \nu(s-t) /\left\{(2 \pi)^{6} \nu^{2}\left(1-\nu^{2}\right)^{2}\right\}$. Since $\mathcal{H}^{0}=k e r L$, then $P_{1} f_{1}$ represents the difference between $f_{1}$ and a sinusoidal function and $\left\|P_{1} f_{1}\right\|^{2}$ measures the departure from the sinusoidal function. We obtain estimates $\hat{\omega}_{1}=3.536 \times 10^{-7}, \hat{\theta}_{1}=419.2$ and $\hat{\theta}_{2}=-1.438 \times 10^{-3}$. Spline estimate of $f_{1}$ and its 95\% Bayesian confidence intervals are shown in Figure 1. Estimates based on the L-spline and kernel methods are essentially the same. The smoothing parameter for the spline model is estimated by the GML method while the window width for the kernel was fixed as in Genton and Hall (2007). Residual sum of squares (RSS) based on L-spline and kernel methods are 923.5 and 925.2 respectively. 
The right panel in Figure 1 shows the projection of $\hat{f}_{1}$ onto the subspace $\mathcal{H}^{1}, P_{1} f_{1}$, and its $95 \%$ Bayesian confidence intervals. The plot indicates that the function $f_{1}$ is not significantly different from a sinusoidal function since $P_{1} f_{1}$ is not significantly different from zero. We also fit a sinusoidal function to the folded data and the estimated sinusoidal function is shown in the middle panel of Figure 1. The sinusoidal function is inside the $95 \%$ Bayesian confidence intervals of the L-spline estimate.

Assuming the periodic shape function can be well modeled by a sinusoidal function, we can investigate evolving amplitude and period nonparametrically. This allows us to check the parametric models in (24) and (25). We first consider the following SNRM

$$
y_{i}=\alpha+\exp \left\{f_{2}\left(t_{i}\right)\right\} \sin \left[2 \pi\left\{\beta+x\left(t_{i}\right)\right\}\right]+\epsilon_{i}, \quad i=1, \cdots, n,
$$

where $\alpha$ is a parameter of the mean, $\exp \left\{f_{2}\left(t_{i}\right)\right\}$ is the amplitude function, $\beta$ is a phase parameter and $x(t)$ is given in (25). Note that the exponential transformation is used to enforce the positive constraint on the amplitude. We model $f_{2}$ using cubic spline model (3). We obtain estimates $\hat{\alpha}=7.20$, $\hat{\beta}=0.38, \hat{\theta}_{1}=417.18$ and $\hat{\theta}_{2}=-1.19 \times 10^{-3}$. RSS is reduced to 881.4. To look at the general pattern of the amplitudes, for those periods with more than five observations based on the folded data, we fit a sinusoidal model for each period and then compute amplitude for each period. These estimated amplitudes are shown in the top panels on Figure 2. Even though model (26) fits data better than model (23) based on RSS, the top left panel shows that the amplitudes are still underestimated. The fit to the real data (not shown) also indicates the same problem.

Finally, we consider the following SNRM

$$
y_{i}=\alpha+\exp \left\{f_{2}\left(t_{i}\right)\right\} \sin \left\{2 \pi f_{3}\left(t_{i}\right)\right\}+\epsilon_{i}, \quad i=1, \cdots, n
$$

where both the amplitude function and period are modeled nonparametrically. We model $f_{2}$ and $f_{3}$ using cubic spline models (3). We obtain estimate $\hat{\alpha}=7.23$. RSS is reduced to 237.7. Estimates of functions $f_{2}$ and $f_{3}$ are shown in the top right and bottom left panels of Figure 2. The confidence intervals for $f_{3}$ are so narrow that they are not distinguishable from the estimate of $f_{3}$. The amplitude function fits data much better which leads to a great reduction of the RSS. To look at the general pattern of the periods, we first identify peaks using the CLUSTER method (Veldhuis and Johnson 1986, Yang, Liu and Wang 2005). Since noises are relatively small, even though it was developed 

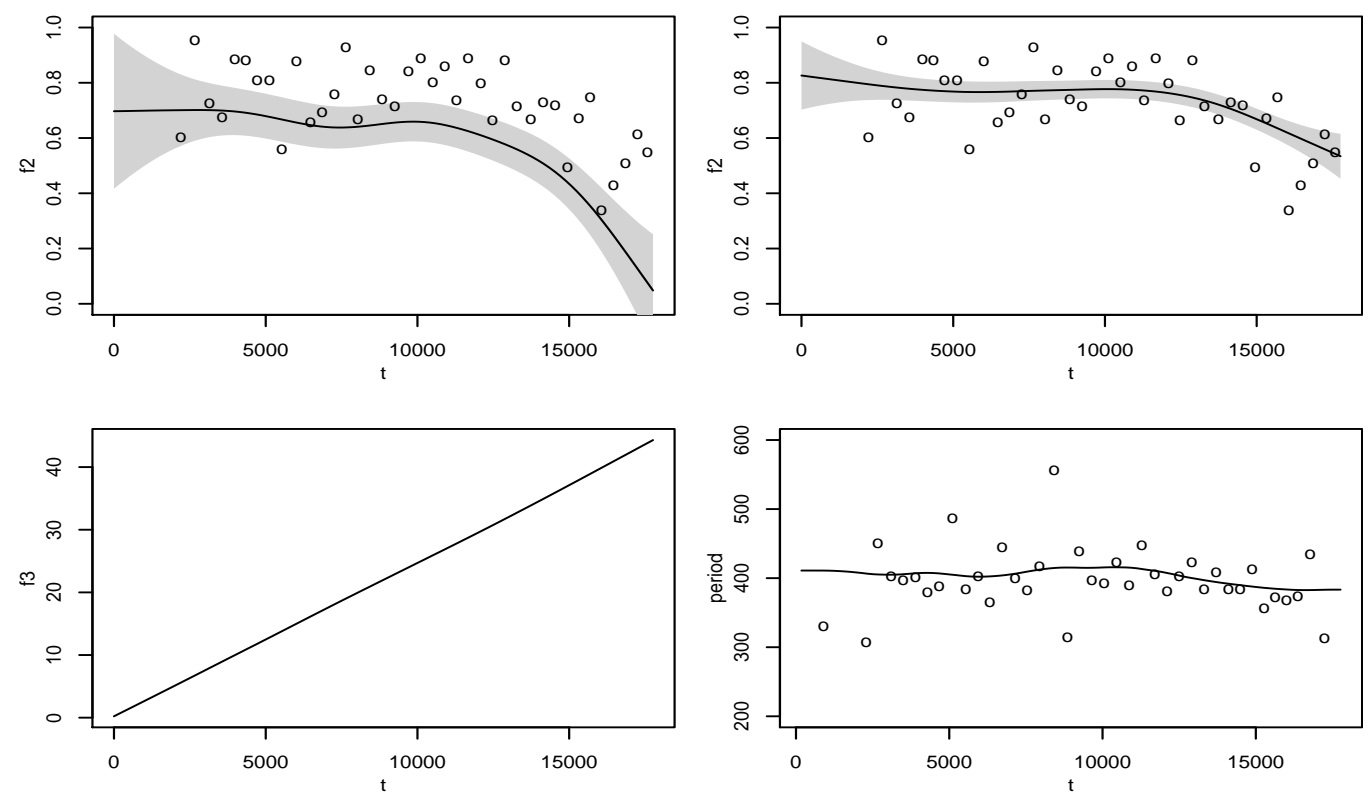

Figure 2: Top: estimated amplitudes based on folded data (circles), spline estimates of $f_{2}$ based on models (26) (left) and (27) (right), and 95\% confidence intervals; bottom left: estimate of $f_{3}$ based on model (27) and its 95\% Bayesian confidence intervals; bottom right: observed periods based on the CLUSTER method (circles) and estimate of the period function $1 / f_{3}^{\prime}(t)$.

for hormone pulse detection, the CLUSTER method identified peaks in the R Hydrae data with few errors. Observed periods are estimated as the lengths between peaks. The bottom right panel of Figure 2 shows the observed periods and the estimate of period function $1 / f_{3}^{\prime}$.

\subsection{Human Growth}

The dataset contains 83 measurements of height of a 10-year-old boy over one school year (Ramsay and Silverman 2002). We are interested in the growth curve over time and its velocity. It is reasonable to assume that the growth curve, $g(t)$, is a monotone increasing function. Let $V(t)=g^{\prime}(t)$ be the velocity function. While there are different ways to fit a monotone function, it is more natural to explicitly enforce the monotonicity by assuming $V(t)>0$. Rewriting $V(t)=\exp \{f(t)\}$, we then can model the function $f$ free of constraint. The growth function $g(t)=V(0)+\int_{0}^{t} V(s) d s$. Therefore, we 
consider the following SNRM

$$
y_{i}=\beta+\int_{0}^{t_{i}} \exp \{f(t)\} d t+\epsilon_{i}, \quad i=1, \cdots, 83
$$

where $y_{i}$ is the height measurement in day $t_{i}$. We model $f$ using the cubic spline (3). For illustration, we consider two error structures: (1) Independence which assumes that $\epsilon_{i}$ 's are iid and (2) AR(1) which assumes that $\epsilon_{i}$ 's follow the first-order autocorrelation structure. Left panel of Figure 3 shows the estimates of the growth curve $g$ under two difference error structures. For comparison, we also fitted a cubic spline for the growth function $g$ without monotone constraint (not shown) and found that parts of the estimate without constraint decrease. The estimate of the auto-correlation coefficient under the $\mathrm{AR}(1)$ error structure is 0.78 which indicates a high auto-correlation. Note that the auto-correlation may be part of the biological signal rather than measurement error. Estimates of the velocity are shown on the right panel of Figure 3. The estimates of the growth curve and velocity under $\mathrm{AR}(1)$ error structure are smoother.
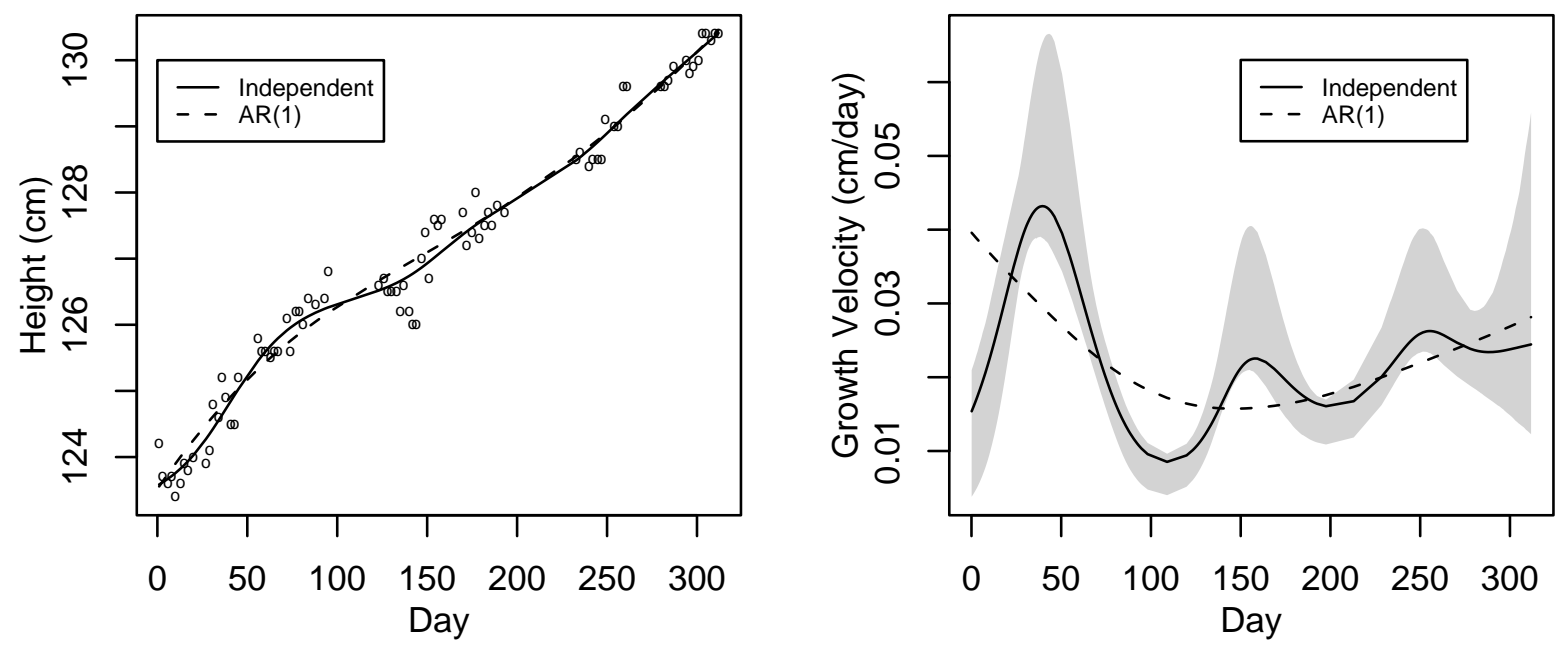

Figure 3: Left: measurements of height (circles) and spline estimates. Right: estimates of growth velocity and $95 \%$ bootstrap confidence intervals for the estimate under the Independence error structure computed using the T-I method in Wang and Wahba (1996) based on 10000 bootstrap samples with random errors sampled with replacement from residuals. 


\subsection{Air Pollution}

The dataset consists of daily measurements of the ozone concentration in parts per million and three meteorological variables: wind speed in miles per hour $\left(x_{1}\right)$, temperature in degrees Fahrenheit $\left(x_{2}\right)$ and solar radiation in Langleys $\left(x_{3}\right)$ for 111 days (Chambers and Hastie 1992). Our goal is to investigate how the air pollutant ozone concentration depends on these three meteorological variables. As pointed out by Yu and Ruppert (2002), a full nonparametric model such as smoothing spline ANOVA decomposition may not be desirable since there are only 111 observations. Yu and Ruppert (2002) considered the following single index model

$$
y_{i}=f_{1}\left(\beta_{1} x_{1 i}+\beta_{2} x_{2 i}+\sqrt{1-\beta_{1}^{2}-\beta_{2}^{2}} x_{3 i}\right)+\epsilon_{i}, \quad i=1, \cdots, 111,
$$

and partially linear single index model

$$
y_{i}=f_{2}\left(\beta_{1} x_{1 i}+\sqrt{1-\beta_{1}^{2}} x_{2 i}\right)+\beta_{2} x_{3 i}+\epsilon_{i}, \quad i=1, \cdots, 111,
$$

where $y_{i}$ is the ozone concentration to the power $1 / 3$ and random errors $\epsilon_{i}$ are iid. Both (29) and (30) are special cases of SNRM. We model $f_{1}$ and $f_{2}$ using the cubic spline (3). The estimate of $f_{1}$ and its $95 \%$ Bayesian confidence intervals are shown in the left panel of Figure 4 . The estimate of $f_{1}$ is similar to that in $\mathrm{Yu}$ and Ruppert (2002). The estimates of $f_{2}$ and $\beta_{2} x_{3 i}$ are shown in the middle and right panels of Figure 4.

The effect of radiation may be non-linear (Ch.7 of Chambers and Hastie (1992)). So we further fit the following SNRM model

$$
y_{i}=f_{3}\left(\beta_{1} x_{1 i}+\sqrt{1-\beta_{1}^{2}} x_{2 i}\right)+f_{4}\left(x_{3 i}\right)+\epsilon_{i}, \quad i=1, \cdots, 111 .
$$

We model $f_{3}$ and $f_{4}$ using the cubic spline (3). For identifiability, the constant basis is removed from the null space $\mathcal{H}^{0}$ for $f_{4}$. Estimates of $f_{3}$ and $f_{4}$ are shown in the middle and right panels of Figure 4. Similar to the estimate in Ch.7 of Chambers and Hastie (1992), $\hat{f}_{4}$ increases with increasing rediation until a value of about 250 Langleys, after which it is flat. The difference between $\hat{f}_{4}$ and the linear estimate is not significant, perhaps due to the small sample size. 

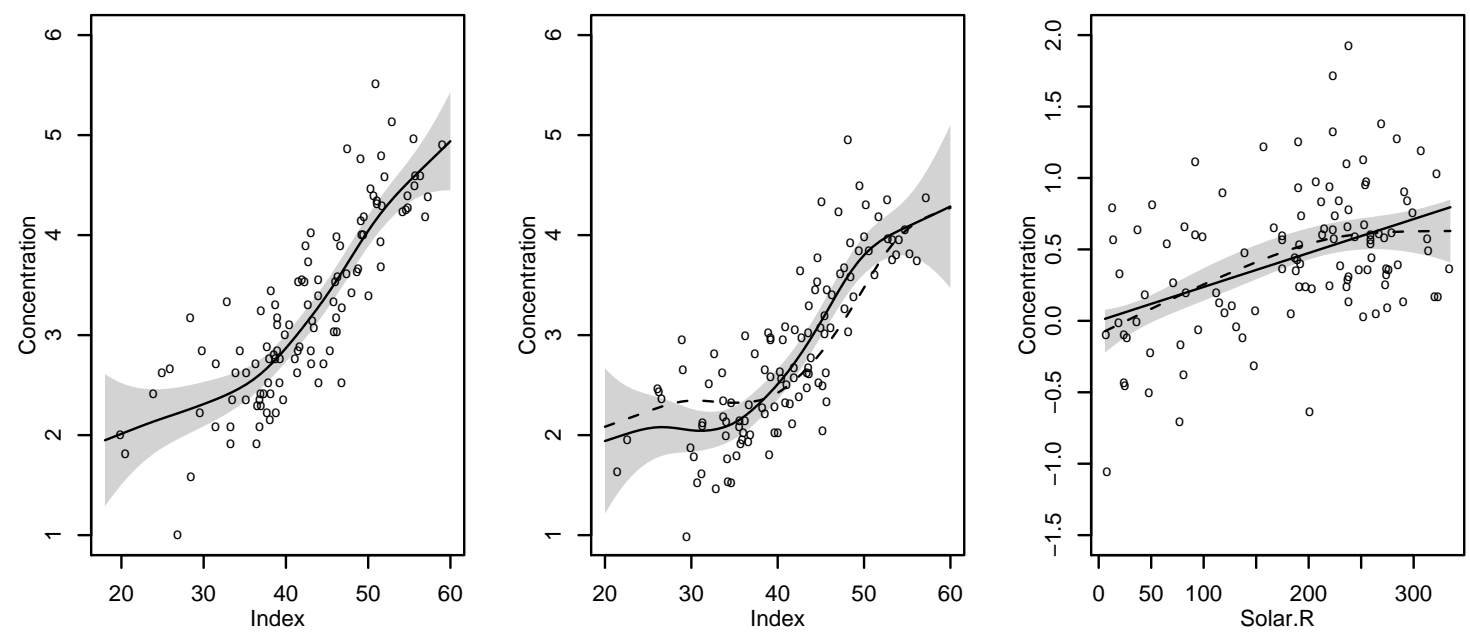

Figure 4: Left: observations $y_{i}$ (circles) and estimate of $f_{1}$ with $95 \%$ Bayesian confidence intervals. Middle: partial residuals after removing rediation effect, estimates of $f_{2}$ (solid line) and $f_{3}$ (dashed line), and 95\% Bayesian confidence intervals for $f_{2}$. Right: partial residuals after removing the index effects based on wind speed and temperature (circles), estimates of $\beta_{2} x_{3 i}$ (solid line) and $f_{4}$ (dashed line), and $95 \%$ Bayesian confidence intervals for $f_{4}$.

\section{Numerical Properties}

We shall evaluate the performance of our methods using simulations. We consider models similar to those in Section 5.1. All simulations are repeated 500 times. Smoothing parameters are estimated by the GML method.

\subsection{Simulation Study with Nonparametric Periodic Shape Function}

Data are generated from model (23), (24) and (25) with $\omega_{1}=1 / n, \theta_{1}=2, \theta_{2}=2 / n, f_{1}(x)=\sin (2 \pi x)$, $t_{i} \stackrel{i i d}{\sim}$ Uniform $[0, n]$ and $\epsilon_{i} \stackrel{i i d}{\sim} \mathrm{N}\left(0, \sigma^{2}\right)$. A factorial design with three different sample sizes $n=$ 250,500, 1000 and three different error standard deviations $\sigma=.05, .20, .40$ is used. The simulation settings are similar to those in Section 3.2 of Genton and Hall (2007). For each simulation sample, we fit model (23), (24) and (25). Table 6.1 lists the biases (e.g. $\left.b\left(\hat{\omega}_{1}\right)=\overline{\hat{\omega}}_{1}-\omega_{1}\right)$, variances and $\operatorname{IMSE}\left(\hat{f}_{1}\right)=\int_{0}^{1}\left\{\hat{f}_{1}(t)-f_{1}(t)\right\}^{2} d t$. Our estimation methods perform as well as or better than that in Genton and Hall (2007). 
Table 1: Biases, variances and IMSEs.

\begin{tabular}{|cccccccc|}
\hline$n$ & $b\left(\hat{\omega}_{1}\right) \times 10^{-6}$ & $b\left(\hat{\theta}_{1}\right) \times 10^{-6}$ & $b\left(\hat{\theta}_{2}\right) \times 10^{-8}$ & $n^{3} \operatorname{var}\left(\hat{\omega}_{1}\right)$ & $n^{3} \operatorname{var}\left(\hat{\theta}_{1}\right)$ & $n^{5} \operatorname{var}\left(\hat{\theta}_{2}\right)$ & $\operatorname{IMSE}\left(\hat{f}_{1}\right)$ \\
\hline$\sigma=.05$ & & & & & & & \\
250 & 0.19 & 1.62 & -1.92 & $6.24 \times 10^{-3}$ & 0.10 & 1.20 & $1.09 \times 10^{-5}$ \\
500 & 0.17 & -2.97 & 2.02 & $5.10 \times 10^{-3}$ & 0.10 & 1.18 & $6.12 \times 10^{-6}$ \\
1000 & 0.06 & -0.15 & 0.06 & $6.43 \times 10^{-3}$ & 0.10 & 1.19 & $2.92 \times 10^{-6}$ \\
$\sigma=.20$ & & & & & & \\
250 & 6.44 & -15.85 & 2.38 & $9.05 \times 10^{-2}$ & 1.66 & 19.94 & $2.06 \times 10^{-4}$ \\
500 & 1.25 & -4.21 & 2.90 & $9.57 \times 10^{-2}$ & 1.75 & 21.40 & $9.17 \times 10^{-5}$ \\
1000 & -0.36 & -1.64 & 0.62 & $9.13 \times 10^{-2}$ & 1.79 & 21.30 & $4.73 \times 10^{-5}$ \\
$\sigma=.40$ & & & & & & & \\
250 & 13.13 & 4.13 & -5.49 & $3.83 \times 10^{-1}$ & 6.10 & 72.73 & $7.35 \times 10^{-4}$ \\
500 & 4.52 & 5.13 & -3.83 & $3.92 \times 10^{-1}$ & 6.50 & 78.54 & $3.86 \times 10^{-4}$ \\
1000 & 0.23 & 2.13 & -0.47 & $3.54 \times 10^{-1}$ & 6.09 & 73.00 & $1.77 \times 10^{-4}$ \\
\hline
\end{tabular}

Left panel of Figure 5 shows the true function of $f_{1}$ and estimates corresponding to the minimum, lower quartile, median, upper quartile and maximum IMSEs when $n=250$ and $\sigma=.2$. Even the worst estimate is very close to the true function.

Left panel of Figure 6 shows the boxplots of coverages of Bayesian and bootstrap confidence intervals when $n=250$ and $\sigma=.2$. Note that coverages of more than half of the replications are $100 \%$, a behavior had been observed before when the true function is in the null space (Gu and Wahba 1993, Wang and Wahba 1995)

\subsection{Simulation Study with Nonparametric Amplitude Function}

Data are generated using the same settings as in subsection 6.1 except the amplitude function. We consider a nonlinear amplitude function $a(t)=\exp \left\{1-.2(t / n)^{4}\right\}$. For each simulation sample, we fit 

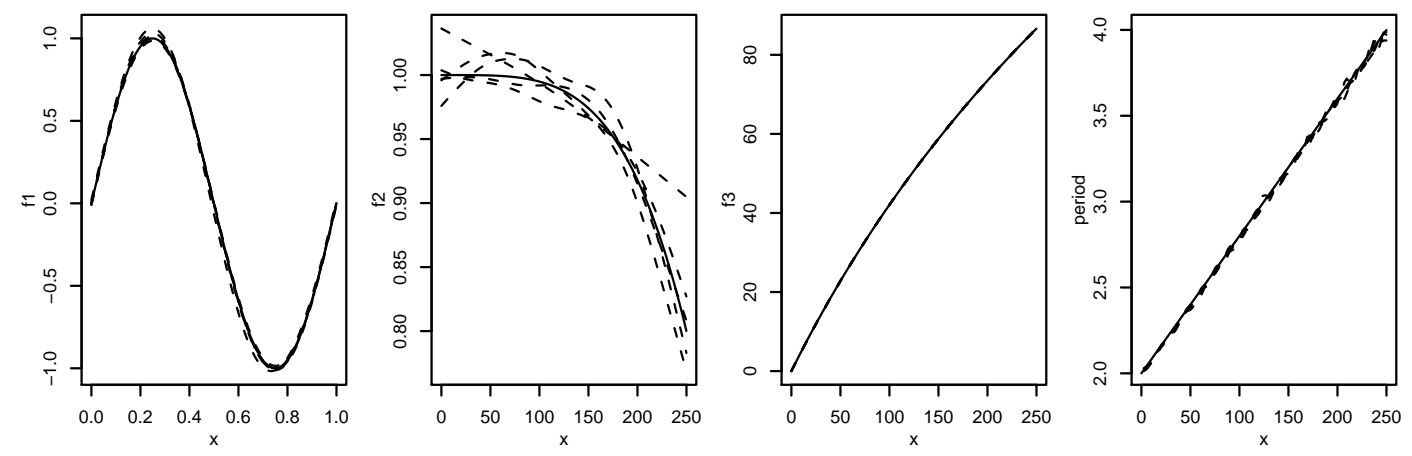

Figure 5: Solid lines in the three left panels are true functions of $f_{1}, f_{2}$ and $f_{3}$ used in subsections 6.1, 6.2 and 6.3 respectively. Solid line in the right panel shows the period function $1 / f_{3}^{\prime}$. Dashed lines are estimates corresponding to the minimum, lower quartile, median, upper quartile and maximum IMSEs when $n=250$ and $\sigma=.2$.
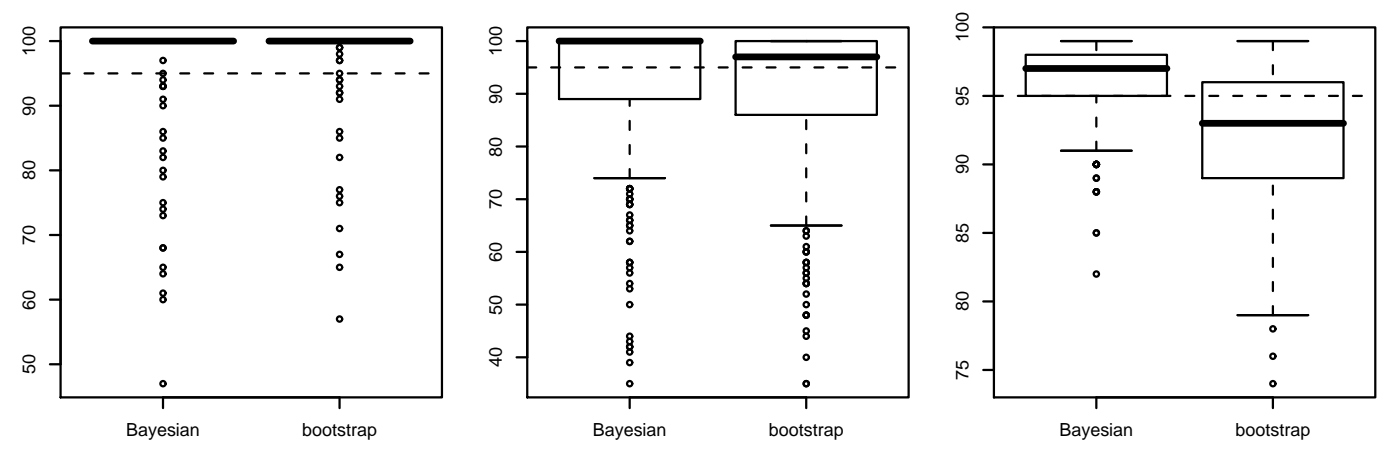

Figure 6: Boxplots of coverages of $95 \%$ Bayesian and bootstrap confidence intervals for functions $f_{1}$ (left), $f_{2}$ (middle) and $f_{3}$ (right). $n=250$ and $\sigma=.2$.

the following model

$$
y_{i}=\exp \left\{f_{2}\left(t_{i}\right)\right\} \sin \left[2 \pi\left\{x\left(t_{i}\right)\right\}\right]+\epsilon_{i}, \quad i=1, \cdots, n,
$$

where $f_{2} \in W_{2}[0, n]$ and $x(t)$ is given in $(25)$. Table 6.2 lists the biases, variances and $\operatorname{IMSE}\left(\hat{f}_{2}\right)=$ $\int_{0}^{n}\left\{\hat{f}_{2}(t)-f_{2}(t)\right\}^{2} d t$. Second panel from the left of Figure 5 shows the true function of $f_{2}$ and estimates corresponding to the minimum, lower quartile, median, upper quartile and maximum IMSEs when $n=250$ and $\sigma=.2$. Middle panel of Figure 6 shows the boxplots of coverages of Bayesian and bootstrap confidence intervals when $n=250$ and $\sigma=.2$. Both estimation and inference methods 
Table 2: Biases, variances and IMSEs.

\begin{tabular}{|cccccc|}
\hline$n$ & $\operatorname{bias}\left(\hat{\theta}_{1}\right)$ & $\operatorname{bias}\left(\hat{\theta}_{2}\right)$ & $n^{3} \operatorname{var}\left(\hat{\theta}_{1}\right)$ & $n^{5} \operatorname{var}\left(\hat{\theta}_{2}\right)$ & $\operatorname{IMSE}\left(\hat{f}_{2}\right)$ \\
\hline$\sigma=.05$ & & & & & \\
250 & $8.53 \times 10^{-7}$ & $-1.24 \times 10^{-8}$ & $3.30 \times 10^{-2}$ & $4.11 \times 10^{-1}$ & $1.99 \times 10^{-5}$ \\
500 & $7.66 \times 10^{-7}$ & $-5.21 \times 10^{-9}$ & $3.41 \times 10^{-2}$ & $4.06 \times 10^{-1}$ & $1.08 \times 10^{-5}$ \\
1000 & $3.19 \times 10^{-7}$ & $-1.59 \times 10^{-9}$ & $3.57 \times 10^{-2}$ & $4.41 \times 10^{-1}$ & $5.92 \times 10^{-6}$ \\
$\sigma=.20$ & & & & \\
250 & $-1.44 \times 10^{-5}$ & $2.12 \times 10^{-7}$ & $6.56 \times 10^{-1}$ & 7.98 & $2.16 \times 10^{-4}$ \\
500 & $3.57 \times 10^{-6}$ & $-2.29 \times 10^{-8}$ & $5.58 \times 10^{-1}$ & 6.79 & $1.14 \times 10^{-4}$ \\
1000 & $-1.57 \times 10^{-6}$ & $5.58 \times 10^{-9}$ & $6.05 \times 10^{-1}$ & 7.50 & $6.53 \times 10^{-5}$ \\
$\sigma=.40$ & & & & & \\
250 & $8.36 \times 10^{-6}$ & $-8.22 \times 10^{-8}$ & 2.38 & 30.02 & $7.97 \times 10^{-4}$ \\
500 & $-9.25 \times 10^{-7}$ & $1.65 \times 10^{-8}$ & 2.63 & 32.30 & $4.25 \times 10^{-4}$ \\
1000 & $1.95 \times 10^{-6}$ & $-8.77 \times 10^{-9}$ & 2.40 & 28.35 & $2.17 \times 10^{-4}$ \\
\hline
\end{tabular}

perform reasonably well.

\subsection{Simulation Study with Nonparametric Periodic Function}

Data are generated using the same settings as in subsection 6.1 except the amplitude function. We consider a nonlinear amplitude function $a(t)=\exp \left\{\omega_{1} t\right\}$ with $\omega_{1}=1 / n$. For each simulation sample, we fit the following model

$$
y_{i}=\exp \left\{\omega_{1} t_{i}\right\} \sin \left[2 \pi\left\{f_{3}\left(t_{i}\right)\right\}\right]+\epsilon_{i}, \quad i=1, \cdots, n,
$$

where $f_{3} \in W_{2}[0, n] \ominus\{1\}$. Table 6.2 lists the biases, variances and $\operatorname{IMSE}\left(\hat{f}_{3}\right)=\int_{0}^{n}\left\{\hat{f}_{3}(t)-f_{3}(t)\right\}^{2} d t$. Second panel from the right of Figure 5 shows the true function of $f_{3}$ and estimates corresponding to the minimum, lower quartile, median, upper quartile and maximum IMSEs when $n=250$ and $\sigma=.2$. The true function and estimates are indistinguishable. The right panel of Figure 5 shows the true periodic function and its estimates. Right panel of Figure 6 shows the boxplots of coverages of 
Table 3: Biases, variances and IMSEs.

\begin{tabular}{|cccc|}
\hline$n$ & $\operatorname{bias}\left(\hat{\omega}_{1}\right)$ & $n^{3} \operatorname{var}\left(\hat{\omega}_{1}\right)$ & $\operatorname{IMSE}\left(\hat{f}_{3}\right)$ \\
\hline$\sigma=.05$ & & & \\
250 & $2.18 \times 10^{-6}$ & $3.80 \times 10^{-3}$ & $1.03 \times 10^{-5}$ \\
500 & $4.99 \times 10^{-7}$ & $3.44 \times 10^{-3}$ & $7.56 \times 10^{-6}$ \\
1000 & $2.26 \times 10^{-7}$ & $2.93 \times 10^{-3}$ & $5.43 \times 10^{-6}$ \\
$\sigma=.20$ & & & \\
250 & $1.20 \times 10^{-5}$ & $6.02 \times 10^{-2}$ & $8.98 \times 10^{-5}$ \\
500 & $4.00 \times 10^{-6}$ & $5.67 \times 10^{-2}$ & $6.61 \times 10^{-5}$ \\
1000 & $1.37 \times 10^{-6}$ & $6.06 \times 10^{-2}$ & $4.76 \times 10^{-5}$ \\
$\sigma=.40$ & & & \\
250 & $3.53 \times 10^{-5}$ & $2.09 \times 10^{-1}$ & $2.60 \times 10^{-4}$ \\
500 & $1.00 \times 10^{-5}$ & $2.02 \times 10^{-1}$ & $1.91 \times 10^{-4}$ \\
1000 & $4.57 \times 10^{-6}$ & $2.37 \times 10^{-1}$ & $1.41 \times 10^{-4}$ \\
\hline & & &
\end{tabular}

Bayesian and bootstrap confidence intervals when $n=250$ and $\sigma=.2$. Both estimation and inference methods perform well.

\section{Conclusion}

The general form of SNRM provides a framework for unified estimation, inference and software development. We focus on methodology and computation in this article. Theoretical properties of our methods are left as future research topics. In addition to fitting special models as illustrated in the article, our methods have other potential applications. For example, the SNRM can be used to check or suggest a nonlinear regression model. Often the problem can be formulated as the hypothesis that $H_{0}: P_{1} f=0$ vs $H_{1}: P_{1} f \neq 0$ (Liu and Wang 2004). One future research topic is to develop methods for testing above hypothesis in SNRMs.

Another potential application of the SNRM is to build model based penalties for spline smoothing. 
The model space $\mathcal{H}$, its decomposition $\mathcal{H}=\mathcal{H}^{0} \oplus \mathcal{H}^{1}$ and the penalty $\left\|P_{1} f\right\|^{2}$ are usually fixed when fitting smoothing spline models (Wahba 1990, Gu 2002). Nevertheless, Heckman and Ramsay (2000) showed that appropriate choice of the model space and its decomposition may involve unknown parameters in the penalty functional. Often, this problem of fitting smoothing spline with unknown parameters in the penalty functional is equivalent to fitting a SNRM.

A closely related field is linear and nonlinear inverse problems. As a result of the interaction of a physical system, a function $f$ is observed through the transformation $T(f)$ where $T$ is a linear or nonlinear operator. The goal is to recover the function $f$ through data. Sometimes the operator $T$ depends on some unknown parameters (Esedoglu 2004, Pillonetto and Bell 2004) which lead to SNRMs. Inverse problems are often ill-posed and regularization is the common approach which is equivalent to the penalized likelihood method when data are observed with random errors. Thus our methods provide an alternative approach for nonlinear inverse problems with data driven choices of regularization parameters.

In this article we are interested in the estimation and inference for both parameters and nonparametric functions. When the parameters are of main interest, for linear semi-parametric models, it is well-known that optimal rate of convergence for parameters can be achieved by using the profile likelihood (Speckman 1988, Severini and Wong 1992, Severini and Staniswalis 1994). Whether these results can be extended to nonlinear semi-parametric models proposed in this article remains an open problem.

\section{References}

Aronszajn, N. (1950). Theory of reproducing kernels, Trans. Amer. Math. Soc. 68: 337-404.

Bates, D. M. and Watts, D. G. (1988). Nonlinear Regression Analysis and Its Applications, Wiley.

Carroll, R. J., Fan, J., Gijbels, I. and Wand, M. P. (1997). Generalized partial linear single-index models, Journal of the American Statistical Association 92: 477-489.

Chambers, J. and Hastie, T. (1992). Statistical Models in S, Wadsworth and Brooks. 
Esedoglu, S. (2004). Blind deconvolution of bar code signals, Inverse Problems 20: 121-135.

Flett, T. M. (1980). Differential Analysis, Cambridge University Press, London.

Friedman, J. H. and Stuetzle, W. (1981). Projection pursuit regression, Journal of the American Statistical Association 76: 817-823.

Genton, M. G. and Hall, P. (2007). Statistical inference for envolving periodic functions, Journal of the Royal Statistical Society B 69: 643-657.

Gu, C. (1989). RKPACK and its applications: Fitting smoothing spline models, Proceedings of the Statistical Computing Section, ASA: pp. 42-51.

Gu, C. (2002). Smoothing Spline ANOVA Models, Springer-Verlag, New York.

Gu, C. and Wahba, G. (1993). Smoothing spline ANOVA with component-wise Bayesian confidence intervals, Journal of Computational and Graphical Statistics 2: 97-117.

Härdle, W., Liang, H. and Gao, J. (2000). Partial linear Models, Springer-Verlag, New York.

Hastie, T. and Tibshirani, R. (1993). Varying coefficient model, Journal of the Royal Statistical Society B 55: $757-796$.

Heckman, N. and Ramsay, J. O. (2000). Penalized regression with model-based penalties, Canadian Journal of Statistics 28: 241-258.

Ke, C. and Wang, Y. (2004). Nonparametric nonlinear regression models, Journal of the American Statistical Association 99: 1166-1175.

Lawton, W. H., Sylvestre, E. A. and Maggio, M. S. (1972). Self-modeling nonlinear regression, Technometrics 13: 513-532.

Liu, A. and Wang, Y. (2004). Hypothesis testing in smoothing spline models, Journal of Statistical Computation and Simulation 74: 581-597.

Mammen, E. and Nielsen, J. P. (2003). Generalised structured models, Biometrika 90: 551-566. 
Pillonetto, G. and Bell, B. M. (2004). Deconvolution of non-stationary physical signals: a smooth variance model for insulin secretion rate, Inverse Problems 20: 367-383.

Pinheiro, J. and Bates, D. M. (2000). Mixed-effects Models in S and S-plus, Springer, New York.

Ramsay, J. O. (1998). Estimating smooth monotone functions, Journal of the Royal Statistical Society B 60: $365-375$.

Ramsay, J. O. and Silverman, B. W. (2002). Applied Functional Data Analysis, Springer, New York.

Ruppert, D., Wand, M. P. and Carroll, R. J. (2003). Semiparametric Regression, Cambridge, New York.

Severini, T. and Staniswalis, J. (1994). Quasi-likelihood estimation in semiparametric models, Journal of the American Statistical Association 89: 501-511.

Severini, T. and Wong, W. (1992). Generalized profile likelihood and conditional parametric models, Annals of Statistics 20: 1768-1802.

Speckman, P. (1988). Kernel-smoothing in partial linear models, Journal of the Royal Statistical Society B 50: 413-436.

Veldhuis, J. D. and Johnson, M. L. (1986). Cluster analysis: a simple versatile and robust algorithm for endocrine pulse detection, American Journal of Physiology 250: E486-E493.

Wahba, G. (1978). Improper priors, spline smoothing, and the problem of guarding against model errors in regression, Journal of the Royal Statistical Society B 40: 364-372.

Wahba, G. (1990). Spline Models for Observational Data, SIAM, Philadelphia. CBMS-NSF Regional Conference Series in Applied Mathematics, Vol. 59.

Wahba, G., Wang, Y., Gu, C., Klein, R. and Klein, B. (1995). Smoothing spline ANOVA for exponential families, with application to the Wisconsin Epidemiological Study of Diabetic Retinopathy, Annals of Statistics 23: 1865-1895. 
Wang, Y. (1998). Smoothing spline models with correlated random errors, Journal of the American Statistical Association 93: 341-348.

Wang, Y. and Brown, M. B. (1996). A flexible model for human circadian rhythms, Biometrics 52: $588-596$.

Wang, Y. and Wahba, G. (1995). Bootstrap confidence intervals for smoothing splines and their comparison to Bayesian confidence intervals, J. Statist. Comput. Simul. 51: 263-279.

Yang, Y., Liu, A. and Wang, Y. (2005). Detecting pulsatile hormone secretions using nonlinear mixed effects partial spline models, Biometrics pp. 230-238.

Yu, Y. and Ruppert, D. (2002). Penalized spline estimation for partially linear single index models, Journal of the American Statistical Association 97: 1042-1054.

Zijlstra, A., Bedding, T. and Mattei, J. (2002). The evolution of the mira variable R Hydrae, Monthly Notices of the Royal Astronomical Society 334: 498-510.

\section{Appendix: Derivation of Posterior Covariances}

Let $\boldsymbol{y}=\boldsymbol{z}+\boldsymbol{\epsilon}$ where $\boldsymbol{z} \sim \mathrm{N}\left(0, b \boldsymbol{\Sigma}_{z, z}\right), \boldsymbol{\epsilon} \sim \mathrm{N}\left(0, \sigma^{2} \boldsymbol{W}^{-1}\right)$ and $\mathrm{E}\left(\boldsymbol{z} \boldsymbol{\epsilon}^{T}\right)=0$. Let $g$ and $h$ be zero mean Gaussian random vectors such that $\mathrm{E}\left(g h^{T}\right)=b \boldsymbol{\Sigma}_{g, h} \mathrm{E}\left(g \boldsymbol{z}^{T}\right)=b \boldsymbol{\Sigma}_{g, z}$ and $\mathrm{E}\left(\boldsymbol{z} h^{T}\right)=b \boldsymbol{\Sigma}_{z, h}$. Let $\sigma^{2} / b=n \lambda$. Then (Gu and Wahba, 1993)

$$
\frac{1}{b} \operatorname{Cov}(g, h \mid \boldsymbol{y})=\boldsymbol{\Sigma}_{g, h}-\boldsymbol{\Sigma}_{g, z}\left(\boldsymbol{\Sigma}_{z, z}+n \lambda \boldsymbol{W}\right)^{-1} \boldsymbol{\Sigma}_{z, h}
$$

Now suppose that we want to compute the posterior covariance between $F_{j}\left(s_{j}\right)$ and $F_{k}\left(t_{k}\right)$. Let $g=F_{j}\left(s_{j}\right), h=F_{k}\left(t_{k}\right)$ and $\boldsymbol{z}=\left(\sum_{k=1}^{r} \mathcal{L}_{k 1} F_{k}, \cdots, \sum_{k=1}^{r} \mathcal{L}_{k n} F_{k}\right)^{T}$. By the correspondence between $\mathcal{H}_{k}^{1}$ and the Hilbert space spanned by $X_{k}\left(t_{k}\right)$ (Wahba 1990, Ch. 1.4), we have

$$
\begin{aligned}
\mathrm{E}\left(\mathcal{L}_{k i} X_{k}\right) X_{k}\left(t_{k}\right) & =\mathcal{L}_{k i(\cdot)} R_{k}^{1}\left(t_{k}, \cdot\right)=\xi_{k i}\left(t_{k}\right), \\
\mathrm{E}\left(\mathcal{L}_{k i} X_{k}\right)\left(\mathcal{L}_{k j} X_{k}\right) & =\mathcal{L}_{k i(\cdot)} \mathcal{L}_{k j(\cdot)} R_{k}^{1} .
\end{aligned}
$$


Let $\boldsymbol{X}_{k}=\left(\mathcal{L}_{k 1} X_{k}, \cdots, \mathcal{L}_{k n} X_{k}\right)^{T}$. Then $\mathrm{E} \boldsymbol{X}_{k} X_{k}\left(t_{k}\right)=\left(\xi_{k 1}\left(t_{k}\right), \cdots, \xi_{k n}\left(t_{k}\right)\right)^{T}=\boldsymbol{\xi}_{k}\left(t_{k}\right)$ and

$$
\begin{aligned}
\mathrm{E}\left(\boldsymbol{z}^{T} \boldsymbol{z}\right) & =b\left(\rho \boldsymbol{T} \boldsymbol{T}^{T}+\boldsymbol{\Sigma}\right), \\
\mathrm{E}(g h) & =\left\{a \sum_{\nu=1}^{m_{k}} \phi_{k \nu}\left(s_{k}\right) \phi_{k \nu}\left(t_{k}\right)+b \theta_{k} R_{k}^{1}\left(s_{k}, t_{k}\right)\right\} I(j=k) \\
& =b\left\{\rho \boldsymbol{\phi}_{k}^{T}\left(s_{k}\right) \boldsymbol{\phi}_{k}\left(t_{k}\right)+\theta_{k} R_{k}^{1}\left(s_{k}, t_{k}\right)\right\} I(j=k), \\
\mathrm{E}\left(g \boldsymbol{z}^{T}\right) & =\mathrm{E}\left\{\boldsymbol{\phi}_{j}^{T}\left(s_{j}\right) \boldsymbol{\zeta}_{j}+\sqrt{b \theta_{j}} X_{j}\left(s_{j}\right)\right\}\left\{\boldsymbol{\zeta}_{j}^{T} \boldsymbol{T}_{j}^{T}+\sqrt{b \theta_{j}} \boldsymbol{X}_{j}^{T}\right\} \\
& =b\left\{\rho \boldsymbol{\phi}_{j}^{T}\left(s_{j}\right) \boldsymbol{T}_{j}^{T}+\theta_{j} \boldsymbol{\xi}_{j}^{T}\left(s_{j}\right)\right\}, \\
\mathrm{E}(\boldsymbol{z} h) & =\mathrm{E}\left\{\boldsymbol{T}_{k} \boldsymbol{\zeta}_{k}+\sqrt{b \theta_{k}} \boldsymbol{X}_{k}\right\}\left\{\boldsymbol{\zeta}_{k}^{T} \boldsymbol{\phi}_{k}\left(t_{k}\right)+\sqrt{b \theta_{k}} X_{k}\left(t_{k}\right)\right\} \\
& =b\left\{\rho \boldsymbol{T}_{k} \boldsymbol{\phi}_{k}\left(t_{k}\right)+\theta_{k} \boldsymbol{\xi}_{k}\left(t_{k}\right)\right\},
\end{aligned}
$$

where $\rho=a / b, \zeta_{k}=\left(\zeta_{k 1}, \cdots, \zeta_{k m_{k}}\right)^{T}$ and $\phi_{k}\left(t_{k}\right)=\left(\phi_{k 1}\left(t_{k}\right), \cdots, \phi_{k m_{k}}\left(t_{k}\right)\right)^{T}$. It is easy to show that formulae (A.4) in Gu and Wahba (1993) hold with $\boldsymbol{M}=\boldsymbol{\Sigma}+n \lambda \boldsymbol{W}^{-1}$ for correlated data. Plugging (A.2) back in (A.1), we then have

$$
\begin{aligned}
& \frac{1}{b} \operatorname{Cov}\left\{F_{j}\left(s_{j}\right), F_{k}\left(t_{k}\right) \mid \boldsymbol{y}\right\} \\
=\quad & \left\{\rho \boldsymbol{\phi}_{k}^{T}\left(s_{k}\right) \boldsymbol{\phi}_{k}\left(t_{k}\right)+\theta_{k} R_{k}^{1}\left(s_{k}, t_{k}\right)\right\} I(j=k) \\
& -\left\{\rho \phi_{j}^{T}\left(s_{j}\right) \boldsymbol{T}_{j}^{T}+\theta_{j} \boldsymbol{\xi}_{j}^{T}\left(s_{j}\right)\right\}\left(\rho \boldsymbol{T} \boldsymbol{T}^{T}+\boldsymbol{M}\right)^{-1}\left\{\rho \boldsymbol{T}_{k} \phi_{k}\left(t_{k}\right)+\theta_{k} \boldsymbol{\xi}_{k}\left(t_{k}\right)\right\} \\
=\quad & \boldsymbol{\phi}_{j}^{T}\left(s_{j}\right)\left\{\rho I(j=k) \boldsymbol{I}-\rho \boldsymbol{T}_{j}^{T}\left(\rho \boldsymbol{T} \boldsymbol{T}^{T}+\boldsymbol{M}\right)^{-1} \rho \boldsymbol{T}_{k}\right\} \phi_{k}\left(t_{k}\right) \\
& -\rho \boldsymbol{\phi}_{j}^{T}\left(s_{j}\right) \boldsymbol{T}_{j}^{T}\left(\rho \boldsymbol{T} \boldsymbol{T}^{T}+\boldsymbol{M}\right)^{-1} \theta_{k} \boldsymbol{\xi}_{k}\left(t_{k}\right) \\
& -\theta_{j} \boldsymbol{\xi}_{j}^{T}\left(s_{j}\right)\left(\rho \boldsymbol{T} \boldsymbol{T}^{T}+\boldsymbol{M}\right)^{-1} \rho \boldsymbol{T}_{k} \boldsymbol{\phi}_{k}\left(t_{k}\right)+\theta_{k} R_{k}^{1}\left(s_{k}, t_{k}\right) I(j=k) \\
& -\theta_{j} \theta_{k} \boldsymbol{\xi}_{j}^{T}\left(s_{j}\right)\left(\rho \boldsymbol{T} \boldsymbol{T}^{T}+\boldsymbol{M}^{-1} \boldsymbol{\xi}_{k}\left(t_{k}\right)\right. \\
\stackrel{\rho \rightarrow \infty}{\rightarrow} & \boldsymbol{\phi}_{j}^{T}\left(s_{j}\right) \boldsymbol{U}_{j k} \boldsymbol{\phi}_{k}\left(t_{k}\right)-\boldsymbol{\phi}_{j}^{T}\left(s_{j}\right) \boldsymbol{V}_{k}\left(t_{k}\right)-\boldsymbol{V}_{j}^{T}\left(s_{j}\right) \boldsymbol{\phi}_{k}\left(t_{k}\right)+\theta_{k} R_{k}^{1}\left(s_{k}, t_{k}\right) I(j=k) \\
& -\theta_{j} \theta_{k} \boldsymbol{\xi}_{j}^{T}\left(s_{j}\right)\left\{\boldsymbol{M}^{-1}-\boldsymbol{M}^{-1} \boldsymbol{T}^{T}\left(\boldsymbol{T}^{T} \boldsymbol{M}^{-1} \boldsymbol{T}\right)^{-1} \boldsymbol{T}^{T} \boldsymbol{M}^{-1}\right\} \boldsymbol{\xi}_{k}\left(t_{k}\right),
\end{aligned}
$$

where $\boldsymbol{U}_{j k}$ is a $m_{j} \times m_{k}$ matrix corresponds to rows $\sum_{l=1}^{j-1} m_{l}+1$ to $\sum_{l=1}^{j} m_{l}$ and columns $\sum_{l=1}^{k-1} m_{l}+1$ to $\sum_{l=1}^{k} m_{l}$ of the matrix $\left(\boldsymbol{T}^{T} \boldsymbol{M}^{-1} \boldsymbol{T}\right)^{-1}$, and $\boldsymbol{V}_{k}\left(t_{k}\right)$ is a subvector of $\left(\boldsymbol{T}^{T} \boldsymbol{M}^{-1} \boldsymbol{T}\right)^{-1} \boldsymbol{T}^{T} \boldsymbol{M}^{-1} \boldsymbol{\xi}_{k}\left(t_{k}\right)$ with elements from $\sum_{l=1}^{k-1} m_{l}+1$ to $\sum_{l=1}^{k} m_{l}$.

Since $g_{j \nu}^{0}\left(s_{j}\right), g_{j}\left(s_{j}\right), g_{k \mu}^{0}\left(t_{k}\right)$ and $g_{k}\left(t_{k}\right)$ in $(21)$ correspond to some components in $F_{j}\left(s_{j}\right)$ and $F_{k}\left(t_{k}\right)$, posterior covariances in $(21)$ can be derived similarly by picking up corresponding components. 